\title{
Delivering care at home through a smarter environment
}

\author{
C.D. Nugent \\ School of Computing and Mathematics, Computer Science Research Institute \\ University of Ulster \\ Shore Road, Newtownabbey \\ Co. Antrim, BT37 0QB \\ cd.nugent@ulster.ac.uk
}

\begin{abstract}
One of the main challenges which society faces as our population continues to grow is the demand which is being placed of both health and social care provision. This is coupled with the need to develop new approaches to allow the general public to take more control of their own health and lifestyle management. The increased prevalence of technology usage, its reduced costs and improved processing and communication capabilities are all key factors which have resulted in new technology based solutions being investigated to address these challenges. Through a consolidation of sensing technology with the ability to record data, intelligent data analysis to interpret the data collected and personalised interfaces to support interaction with users, it has now become possible to deliver support both when and where it is required. The aim of this paper is to provide an overview of the technological motivations and solutions which have been developed to support home based care. Specifically the work presented will discuss the challenges associated with managing the data generated within a smart environment and from a technology perspective consider what are the future trends within the domain.
\end{abstract}

Home based care, assistive technologies, behavioural analysis, smart homes, smart environments, information management.

\section{INTRODUCTION}

It is a recognised fact that the world is rapidly ageing. According to projections from the United Nations, estimates suggest that the world's population will increase to close to 8.9 billion within the next 40 years. This equates to close to a $47 \%$ increase since the year 2000. Within nearly every country across the globe, the age group of those aged 60 and over is the fastest growing. The main contributing factors to this growth can be attributed to increased life expectancy and a decrease in fertility rates. According to the World Health Organisation (WHO), by 2050, the number of older people on a global scale will have increased to 2 billion. This is an increase of nearly 3 fold, given the figure of 600 million older people in the year 2000 [1].

The advances from economic, social and medical perspectives can be viewed as the main contributing factors to global ageing. Nevertheless, the consequence of an ageing society has resulted in many challenges in the form of increased demands being placed on health and social care along with burdens being placed on both social insurance and pension schemes. The WHO have, at a general level, identified a number of areas where effort should be directed in an effort to alleviate some of the aforementioned challenges:

- Healthy ageing and prevention of chronic disease.

- Improvements in the process related to management of chronic disease.

- $\quad$ Creation of environments which can be recognized as being age friendly.

Taking all of this into consideration it is evident that there are benefits to be gained by addressing the challenges which are presenting themselves from an ageing population. Although not being claimed as the definitive solution, the use of technology is being widely recognised as having a significant role to play. Its increased prevalence, reduced costs and improved processing and communication speeds are all key factors which have resulted in new technology based solutions being investigated.

This paper aims to provide an insight into the motivations behind the introduction of technology based home care. In addition, consideration will be given to the complexities of managing data from heterogeneous sources along with an insight into expected trends for the future. The remainder of the paper is structured as follows. In Section II an overview of a number of technological concepts and architectures is presented. This is extended in Section III when the notion of a smart home is introduced along with the types of information which can be monitored. Section IV considers the trends within this area and introduces a number of potential areas where improvement in the overall process of providing delivery of care at home may be targeted. The paper is concluded in Section V.

\section{TECHNOLOGY AND HEALTHCARE}

Technology has been used within medicine for an extended period of time. Augustus D. Waller is recognised as the first person to record the electrical signals from the heart using a mercury capillary electrometer in 1887 [2]. Einthoven extended the work of Waller and developed the string galvanometer in 1903. Hans Berger made one of the first recordings of the electrical signals produced by the human brain in 1924 and began the process of identifying normal and 
abnormal brain waves. The usage of computerized techniques to process electrocardiograms by Pipberger in 1957 is considered to be the first instance of computers being used in medicine [3]. Since then, over a period of slightly more than 50 years, computer based technology has embedded itself firmly within the provision and management of healthcare extending hospital, community and home based settings. This has been largely influenced by advances in computing technology, the Internet and both fixed and wireless modes of communications.

Although there have been many efforts to define terminology along with presentation of high level strategies and impactful aims of how technology should be used to support home based care, the Ambient Assisted Living (AAL) Program has encapsulated the key aspects which should be taken into consideration [4, 5]. Through their consolidated efforts a Program of Work has been established which has recognised that there is an opportunity to improve the quality of life of older people through the use of Information and Communication Technologies (ICT). This requires the combination of products, services and systems that together have the potential to create smarter environments at home, at work and in the community. Figure 1 depicts what may be interepreted as the main factors which contribute to the AAL Program.

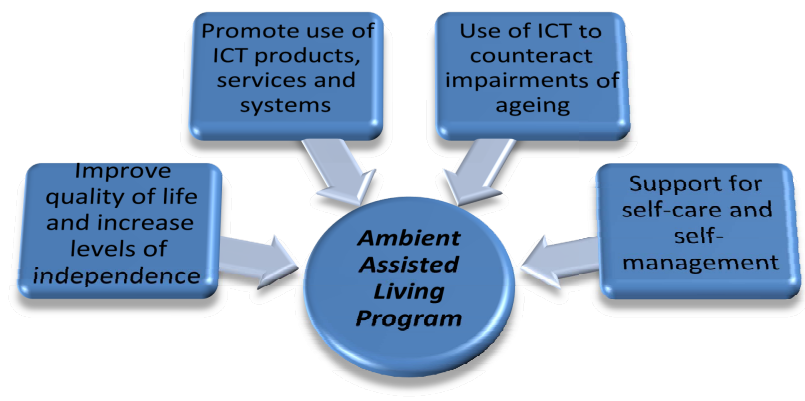

Figure 1. Overview of the main factors contributing to the Ambient Assisted Living Program [4, 5].

\section{THE SMART HOME PARADIGM}

A number of home based assistive technologies have been successfully introduced which can offer improved levels of independence for example medication management devices, devices to acquire vital signs, cognitive prosthetics, to name but a few [6]. There is, however, benefit to consider more information than that which is provided by a single device working in isolation. This provides an opportunity to create a smarter living environment and is in accordance with the visions of the AAL Program of using ICT products, services and systems in an effort to counteract the impairments associated with ageing.

If we consider the smart home paradigm, in its most general sense, we can identify it as having three main components (refer to Figure 2):
1. Sensors. These are the devices which are installed and seamlessly embedded within the home and can collect information as people undertake their daily activities. Examples of sensor based technologies include motion sensors, video capturing and the recording of basic vital signs.

2. Data processing. This component is responsible for the processing of the data collected in an effort to detect either changes in behavior over time or identifying that assistance is required at a specific point in time.

3. Environment Control. Based on the output of the previous stage of data processing various forms of interactive technology can be used to either control the environment or provide feedback to the person within their environment.

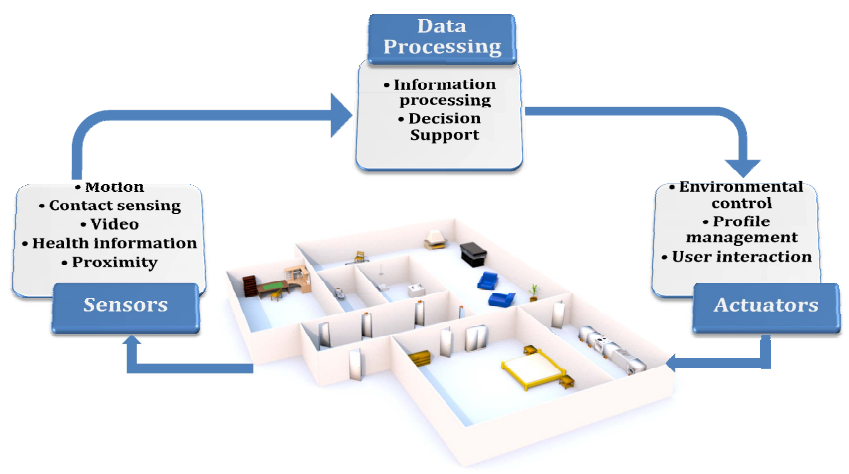

Figure 2. The Smart Home Paradigm with the three main components of Sesning Technology, Data Processing and Environment control.

The area of research within this domain has attracted a huge amount of interest from academic, industrial and clinical perspectives. A number of demonstrator environments are currently in place along with a range of larger scale environments which are aiming to provide real living environments to support ageing in place [7, 8]. Although these environments are in existence, their provision of home based care has not reached a level of providing a framework which supports all elements of required support which can be tailored on a case by case basis. As such, it is very much an active area of research with a number of key challenges requiring further consideration.

\section{CHALLENGES AND OPPORTUNITIES}

As previously discussed, although significant progress has been made within the domain of smart environments, one of the key outstanding issues in the delivery of an efficient form of care provision at home is the appropriate management and processing of the heterogeneous data generated from the sensor technology. From a sensing perspective a number of parameters can be acquired which can provide information in relation to the health and well being profile of the person within their home. This information can then be used to either provide a form of real time assistance or, following assessment over long periods of data collection, deliver warnings in instances of cognitive or physical decline.

General health status can be assessed through usage of a number of off the shelf technologies which can be integrated 
into home based networks through either fixed or wireless communication means. Assessment of activities within the environment can be captured through wearable technologies with inbuilt accelerometers for example mobile phones or can be captured more subtly through interaction with everyday devices such as the Nintendo Wii. Assistive technologies such as cognitive prosthetics, medication management devices and fall detectors can all relay information to a central processing unit within the home. In addition, a user's interaction with the environment for example opening doors and lifting objects, along with motion sensors detecting spatial orientation, can provide information which can support behavioural analysis. As depicted in Figure 3, based on the available information, analysis can be provided at two levels. In the first instance coarse grained analysis can provide an understanding in relation to a person being active or not, or that a specific discrete vital sign measurement is within or out of range. On the other hand, a finer grained analysis of the information collected can provide an insight into the type of activity. For example pacing, wandering in a room or analysis of object interaction can provide specific details of which stage a person is currently at in terms of completing a task.

The real benefit of all of these sensing technologies, and perhaps the key opportunity within the management of information within the smart home paradigm, is the consideration of how they can be used to augment the overall information management process. For example, a contact sensor can be used to detect that someone has opened a fridge, however, if there are two people in the environment it will be unclear which person it is. If the underlying information management engine were to be augmented with rudimentary video processing information with the capability to differentiate between two people based on their body shape or clothing colours, then it becomes possible to detect not only which object has been interacted with, but also which of the two people it actually was.

There exists a trend at present where the level of information processing is now moving beyond that of considering information from one device in isolation to a framework which can manage and process heterogeneous information sources. As a result, more elaborate service delivery will become possible which will have the capability to address practical challenges in managing care within smart environments.

\section{CONCLUSION}

It is evident that technology has a role to play in offering improvements in home based care management. The creation of a smart environment within the home has the ability to not only offer an opportunity to embed sensors to acquire information about a person within their own home, it also provides the opportunity to process the information and subsequently interact with the person with the aim of enhancing the underlying quality of life.

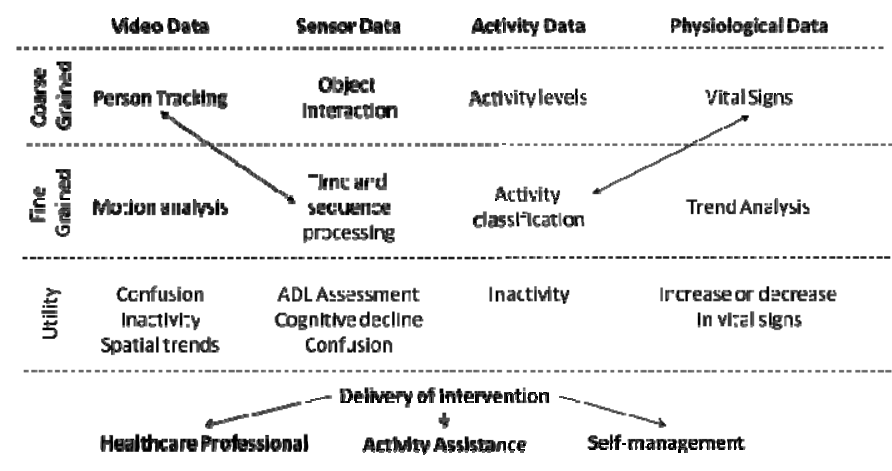

Figure 3. Augmentation of information collected within the smart home environment.

To date, the impact of research conducted within the domain has provided a deeper insight into the factors associated with the design and development of technology to support independent living. This has the benefit of being available to inform further work in this area. Many challenges currently exist which are at present hindering the large scale rollout and uptake of smart environments. Within this paper, the concept of improving the overall process of information management has been considered through a framework of combining heterogeneous data. Although this may be viewed as only one component of a much larger challenge, it is the author's view that such incremental advances can in the long term have a much more significant effect.

\section{REFERENCES}

[1] Ageing, World Health Organistion. http://www.who.int/topics/ageing/en/

[2] A. D. Waller, A demonstration on man of electromotive changes accompanying the hearts beat, Journal of Physiology, vol. 8, no. 5, pp. 229-234, 1887.

[3] J. D. Bronzino, The Biomedical Engineering Handbook, New York, CRC Press, 1999.

[4] Ambient Assisted Living, COM (2007) 329 Final, pp.1-35, 2007.

[5] The Ambient Assisted Living (AAL) Joint Programme (http://www.aaleurope.eu).

[6] C. D. Nugent, D. D. Finlay, P. Fiorini, Y. Tsumaki, E. Prassler, Home automation as a means of independent living, IEEE Transactions on Automation Science and Engineering, vol. 5, no. 1, pp. 1-9, 2008.

[7] D. J. Cook and S. J. Das, How smart are our environments? An updated look at the state of the art, Journal of Pervasive and Mobile computing, vol. 3, pp. 53-73, 2007.

[8] M. P. Poland, C. D. Nugent, H. Wang, L. Chen, Smart Home Research: Projects and Issues, The International Journal of Ambient Computing and Intelligence, vol. 1, no. 4, pp. 32-45, 2009. 